\title{
On approximating Mills ratio
}

\section{Zhen-Hang Yang and Yu-Ming Chu*}

"Correspondence: chuyuming2005@126.com School of Mathematics and Computation Science, Hunan City University, Yiyang, 413000, China

\begin{abstract}
In the article, we present several sharp bounds for the Mills ratio $R(x)=e^{x^{2} / 2} \int_{x}^{\infty} e^{-t^{2} / 2} d t(x>0)$ in terms of the functions $I_{a}(x)=a /\left[\sqrt{x^{2}+2 a}+(a-1) x\right]$ and $J(x)=a /\left[\sqrt{x^{2}+2 a^{2} / \pi}+2 a x / \pi\right]$ with parameter $a>0$.
\end{abstract}

MSC: $60 \mathrm{E} 15 ; 26 \mathrm{~A} 48 ; 26 \mathrm{D} 15$

Keywords: Mills ratio; monotonicity; convexity; bound

\section{Introduction}

The Mills ratio [1] is the function

$$
R(x)=\frac{1-\Phi(x)}{\phi(x)}=e^{\frac{x^{2}}{2}} \int_{x}^{\infty} e^{-\frac{t^{2}}{2}} d t, \quad x>0,
$$

where $\phi(x)=e^{-x^{2} / 2} / \sqrt{2 \pi}$ is the density function of a standard Gaussian law and $\Phi(x)=$ $\int_{-\infty}^{x} \phi(t) d t$ its cumulative distribution function. The study of the Mills ratio is much older than the work of Mills [1], and through its relation with the function $F(x)=e^{x^{2}} \int_{x}^{\infty} e^{-t^{2}} d t$ given by $R(x)=\sqrt{2} F(x / \sqrt{2})$, its introduction can be traced back to Laplace [2], Livre X, Chapter $1, \mathrm{n}^{\circ} 5$, while he was analyzing different hypotheses related to the refraction of the light in the atmosphere. Laplace gave many of the essential results, like the continued fraction and the asymptotic expansion. Since the function $F$ is related to the error function, and also to the upper incomplete Gamma function of parameter $1 / 2$, the properties of the Mills ratio are spread over papers and books of probability and statistics, mathematical analysis, numerical analysis, etc., and many results have been discovered and rediscovered by different authors.

It is well known that the function $\Phi$ cannot be expressed as the composition of elementary functions, therefore, it is valuable to find sharp bounds for the Mills ratio by certain simple and elementary functions.

Gordon [3] proved that the double inequality

$$
\frac{x}{x^{2}+1} \leq R(x) \leq \frac{1}{x}
$$

holds for all $x>0$.

Birnbaum [4] and Komatu [5] proved that the double inequality

$$
\frac{2}{\sqrt{x^{2}+4}+x}<R(x)<\frac{2}{\sqrt{x^{2}+2}+x}
$$

holds for all $x>0$.

(C) 2015 Yang and Chu. This article is distributed under the terms of the Creative Commons Attribution 4.0 International License (http://creativecommons.org/licenses/by/4.0/), which permits unrestricted use, distribution, and reproduction in any medium, provided you give appropriate credit to the original author(s) and the source, provide a link to the Creative Commons license, and indicate if changes were made. 
An improvement for the upper bound of Mills ratio,

$$
R(x)<\frac{2}{\sqrt{x^{2}+8}+3 x}
$$

is due to Sampford [6] and Shenton [7].

Pollak [8] proved that $b=4 / \pi$ is the best possible parameter such that $2 /\left[\sqrt{x^{2}+b}+x\right]$ is the upper bound of Mills ratio. Boyd [9] dealt with the bounds for the Mills ratio of the form

$$
\psi(x)=\frac{a}{\sqrt{x^{2}+b}+c}
$$

with $a, b, c>0$ such that

$$
\psi(0)=R(0), \quad \lim _{x \rightarrow \infty}[x(\psi(x)-R(x))]=0
$$

and proved that

$$
\frac{\pi}{\sqrt{x^{2}+2 \pi}+(\pi-1) x}<R(x)<\frac{\pi}{\sqrt{(\pi-2)^{2} x^{2}+2 \pi x}+2 x} .
$$

Very recently, Gasull and Utzet [10] proved the double inequality

$$
\max \left\{W_{3,0}(x), W_{1,2}(x)\right\}<R(x)<\max \left\{W_{0,3}(x), W_{2,1}(x)\right\}
$$

for all $x>0$, where

$$
\begin{array}{ll}
W_{3,0}(x)=\frac{\pi}{\sqrt{2(4-\pi) x^{2}+2 \pi}+2 x}, & W_{1,2}(x)=\frac{\pi}{\sqrt{x^{2}+2 \pi}+(\pi-1) x}, \\
W_{2,1}(x)=\frac{\pi}{\sqrt{(\pi-2)^{2} x^{2}+2 \pi}+2 x}, & W_{0,3}(x)=\frac{4}{\sqrt{x^{2}+8}+3 x} .
\end{array}
$$

More inequalities involving the Mills ratio $R(x)$ can be found in the literature [11-21] and the references therein.

Let $\psi(x)$ be defined by (1.2). Then making use of the asymptotic expansion of the Mills ratio $R(x)$ at infinity (see [22], p. 44)

$$
R(x) \sim \frac{1}{x}-\frac{1}{x^{3}}+\frac{1 \cdot 3}{x^{5}}-\frac{1 \cdot 3 \cdot 5}{x^{7}}+\cdots \quad(x \rightarrow \infty)
$$

we get

$$
\psi(x)=\frac{a}{\sqrt{x^{2}+2 a}+(a-1) x}:=I_{a}(x)
$$

if $\psi(x)$ satisfies

$$
\lim _{x \rightarrow \infty}[x(\psi(x)-R(x))]=0, \quad \lim _{x \rightarrow \infty}\left[x^{3}(\psi(x)-R(x))\right]=0,
$$


and

$$
\psi(x)=\frac{a}{\sqrt{x^{2}+2 a^{2} / \pi}+2 a x / \pi}:=J_{a}(x)
$$

if $\psi(0)=R(0)$ and $\psi^{\prime}(0)=R^{\prime}(0)$.

The main purpose of this paper is to present the sharp bounds for the Mills ratio $R(x)$ in terms of $I_{a}(x)$ and $J_{a}(x)$.

\section{Lemmas}

In order to prove our main results we need several lemmas, which we present in this section.

Lemma 2.1 (See [23], Proposition 1.1 or [24], Proposition 1.2) Let $-\infty \leq a<b \leq \infty, f, g$ : $(a, b) \rightarrow \mathbb{R}$ be differentiable on $(a, b)$ with $f\left(a^{+}\right)=g\left(a^{+}\right)=0$ or $f\left(b^{-}\right)=g\left(b^{-}\right)=0$, and $g^{\prime}(x) \neq$ 0 on $(a, b)$. If $f^{\prime}(x) / g^{\prime}(x)$ is increasing (decreasing) on $(a, b)$, then so is $f(x) / g(x)$.

Lemma 2.2 (See [25], Theorem 9) Let $-\infty \leq a<b \leq \infty, f, g:(a, b) \rightarrow \mathbb{R}$ be differentiable on $(a, b)$ with $f\left(b^{-}\right)=g\left(b^{-}\right)=0, g^{\prime}(x) \neq 0$ on $(a, b)$, and

$$
H_{f, g}=\frac{f^{\prime}}{g^{\prime}} g-f \text {. }
$$

If there exists $c \in(a, b)$ such that $f^{\prime} / g^{\prime}$ is increasing (decreasing) on $(a, c)$ and decreasing (increasing) on $(c, b)$. Then the follows statements are true:

(i) $\mathrm{f} / \mathrm{g}$ is decreasing (increasing) on $(a, b)$ if $g^{\prime}>0$ on $(a, b)$ and $H_{f, g}\left(a^{+}\right) \leq(\geq) 0$ or $g^{\prime}<0$ on $(a, b)$ and $H_{f, g}\left(a^{+}\right) \geq(\leq) 0$;

(ii) there exists $c_{0} \in(a, b)$ such that $f / g$ is increasing (decreasing) on $\left(a, c_{0}\right)$ and decreasing (increasing) on $\left(c_{0}, b\right)$ if $g^{\prime}>0$ on $(a, b)$ and $H_{f, g}\left(a^{+}\right)>(<) 0$ or $g^{\prime}<0$ on $(a, b)$ and $H_{f, g}\left(a^{+}\right)<(>) 0$.

Lemma 2.3 Let $a, b \in \mathbb{R}$ with $a<b$ and $f:[a, b] \rightarrow \mathbb{R}$ be continuous and strictly convex (concave) with $f(a) f(b)<0$. Then there exists $c \in(a, b)$ such that $f(x) f(a)>0$ for $x \in(a, c)$ and $f(x) f(b)>0$ for $x \in(c, b)$.

Proof We only prove the case of $f$ being convex with

$$
f(a)<0, \quad f(b)>0,
$$

other cases can be proved by similar methods. Let $x \in(a, b)$ and

$$
F(x)=\frac{f(x)-f(a)}{x-a}
$$

Then from the convexity of $f$ on $[a, b]$ we know that $F$ is increasing on $(a, b)$.

We divide the proof into two cases.

Case $1 F\left(a^{+}\right) \geq 0$. Then $F(x)>F\left(a^{+}\right) \geq 0$ for $x \in(a, b)$. It follows from the convexity of $f$ on $[a, b]$ that 


$$
\frac{f\left(x_{2}\right)-f\left(x_{1}\right)}{x_{2}-x_{1}}>\frac{f\left(x_{2}\right)-f(a)}{x_{2}-a}=F\left(x_{2}\right)>0
$$

for all $x_{1}, x_{2} \in(a, b)$ with $x_{1} \neq x_{2}$.

Inequality (2.4) implies that $f$ is increasing on $(a, b)$, which together with (2.2) leads to the desired result.

Case $2 F\left(a^{+}\right)<0$. Then from the monotonicity of $F$ given by (2.3) we clearly see that $F(b)>0$ and there exists $c^{*} \in(a, b)$ such that $F(x)<0$ for $x \in\left(a, c^{*}\right)$ and $F(x)>0$ for $x \in$ $\left(c^{*}, b\right)$.

From (2.2) and (2.3) together with Case 1 we know that

$$
f(x)<f(a)<0
$$

for $x \in\left(a, c^{*}\right)$ and $f$ is increasing on $\left(c^{*}, b\right)$.

Therefore, the desired assertion follows from (2.5) and the monotonicity of $f$ on $\left(c^{*}, b\right)$ together with (2.2).

Lemma 2.4 Let

$$
h(t)=-2 \pi a t^{3}-3 \pi^{2} t^{2}+2 a\left(2 a^{2}-3 \pi\right) t-\left[2(4-\pi) a^{2}-\pi^{2}\right] .
$$

Then the equation $h^{\prime}(t)=0$ has the unique solution

$$
t_{0}=t_{0}(a)=\frac{\sqrt{8 a^{4}-12 \pi a^{2}+3 \pi^{3}}-\sqrt{3 \pi} \pi}{2 \sqrt{3 \pi} a}
$$

on $(0,1)$ such that $h\left(t_{0}\right)>0$ if $a \in(\pi / \sqrt{2(4-\pi)}, \sqrt{\pi+1}+1)$.

Proof It follows from (2.6) that

$$
\begin{aligned}
& h^{\prime}(t)=-6 \pi a t^{2}-6 \pi^{2} t+2 a\left(2 a^{2}-3 \pi\right), \\
& h^{\prime}(0)=2 a\left(2 a^{2}-3 \pi\right)>0 .
\end{aligned}
$$

From (2.8) and (2.9) we know that $t_{0}=t_{0}(a)$ given by (2.7) is the unique positive solution of the equation $h^{\prime}(t)=0$. Equation (2.7) gives

$$
\frac{d t_{0}(a)}{d a}=\frac{\sqrt{3 \pi} \pi \sqrt{8 a^{4}-12 \pi a^{2}+3 \pi^{3}}+\left(8 a^{4}-3 \pi^{3}\right)}{2 \sqrt{3 \pi} a^{2} \sqrt{8 a^{4}-12 \pi a^{2}+3 \pi^{3}}}>0
$$

for $a \in(\pi / \sqrt{2(4-\pi)}, \sqrt{\pi+1}+1)$.

Inequality (2.10) leads to

$$
t_{0}(a)<t_{0}(\sqrt{\pi+1}+1)<t_{0}\left(\frac{10}{3}\right)=0.7857 \cdots<1
$$

for $a \in(\pi / \sqrt{2(4-\pi)}, \sqrt{\pi+1}+1)$. 
Next, we prove that $h\left(t_{0}\right)>0$ for $a \in(\pi / \sqrt{2(4-\pi)}, \sqrt{\pi+1}+1)$. From (2.6) and (2.7) we have

$$
h\left(t_{0}\right)=\frac{\left(8 a^{4}-12 \pi a^{2}+3 \pi^{3}\right)^{3 / 2}-3 \sqrt{3 \pi}\left(\pi^{2}-4 a^{2}\right)^{2}}{6 \sqrt{3 \pi} a^{2}} .
$$

It is enough to prove that

$$
\begin{aligned}
\left(8 u^{2}\right. & \left.-12 \pi u+3 \pi^{3}\right)^{3}-\left[3 \sqrt{3 \pi}\left(4 u-\pi^{2}\right)^{2}\right]^{2} \\
= & 4 u\left[128 u^{5}-576 \pi u^{4}+144 \pi\left(\pi^{2}+6 \pi-12\right) u^{3}\right. \\
& \left.-432 \pi^{3}(\pi-3) u^{2}-54 \pi^{5}(6-\pi) u+27 \pi^{7}\right] \\
:= & 4 u h_{1}(u)>0
\end{aligned}
$$

for $u \in\left(\pi^{2} /[2(4-\pi)],(\sqrt{\pi+1}+1)^{2}\right)$.

Let $v=u-\pi^{2} /[2(4-\pi)]>0$. Then $h_{1}(u)$ can be rewritten as

$$
\begin{aligned}
h_{1}(u)= & \frac{2}{(4-\pi)^{5}}\left[64(4-\pi)^{5} v^{5}+64 \pi(4-\pi)^{4}(7 \pi-18) v^{4}\right. \\
& +8 \pi(4-\pi)^{3}\left(9 \pi^{4}+74 \pi^{3}-684 \pi^{2}+1,728 \pi-1,728\right) v^{3} \\
& +4 \pi^{3}(4-\pi)^{2}\left(81 \pi^{4}-736 \pi^{3}+2,916 \pi^{2}-6,048 \pi+5,184\right) v^{2} \\
& +\pi^{5}(4-\pi)\left(27 \pi^{5}-324 \pi^{4}+2,000 \pi^{3}-7,272 \pi^{2}+13,824 \pi-10,368\right) v \\
& \left.+4 \pi^{7}\left(9 \pi^{2}-40 \pi+48\right)(\pi-3)^{2}\right]>0
\end{aligned}
$$

due to all the coefficients of the quintic polynomial being positive.

Lemma 2.5 Let $h(t)$ be defined by (2.6). Then the following statements are true:

(i) there exists $t_{1} \in(0,1)$ such that $h(t)>0$ for $t \in\left(0, t_{1}\right)$ and $h(t)<0$ for $t \in\left(t_{1}, 1\right)$ if $a \in(0, \pi / \sqrt{2(4-\pi)}]$

(ii) there exists $t_{11}, t_{12} \in(0,1)$ with $t_{11}<t_{12}$ such that $h(t)<0$ for $t \in\left(0, t_{11}\right) \cup\left(t_{12}, 1\right)$ and $h(t)>0$ for $t \in\left(t_{11}, t_{12}\right)$ if $a \in(\pi / \sqrt{2(4-\pi)}, \sqrt{\pi+1}+1)$;

(iii) there exists $t_{1}^{*} \in(0,1)$ such that $h(t)<0$ for $t \in\left(0, t_{1}^{*}\right)$ and $h(t)>0$ for $t \in\left(t_{1}^{*}, 1\right)$ if $a \in[\sqrt{\pi+1}+1, \infty)$.

Proof It follows from (2.6) that

$$
\begin{aligned}
h(0) & =\pi^{2}-2(4-\pi) a^{2}=-2(4-\pi)\left(a+\frac{\pi}{\sqrt{2(4-\pi)}}\right)\left(a-\frac{\pi}{\sqrt{2(4-\pi)}}\right), \\
h(1) & =4 a^{3}-2(4-\pi) a^{2}-8 \pi a-2 \pi^{2} \\
& =2(\pi+2 a)(a+\sqrt{\pi+1}-1)[a-(\sqrt{\pi+1}+1)], \\
h^{\prime}(t) & =-6 \pi a t^{2}-6 \pi^{2} t+2 a\left(2 a^{2}-3 \pi\right), \\
h^{\prime}(0) & =2 a\left(2 a^{2}-3 \pi\right)=4 a\left(a+\frac{\sqrt{6 \pi}}{2}\right)\left(a-\frac{\sqrt{6 \pi}}{2}\right), \\
h^{\prime}(1) & =4 a^{3}-12 \pi a-6 \pi^{2} .
\end{aligned}
$$


From (2.14) we clearly see that $h^{\prime}(t)$ is decreasing and $h(t)$ is strictly concave on $(0,1)$. We divide the proof into five cases.

Case $1 a \in(0, \pi / \sqrt{2(4-\pi)})$. Then (2.12) and (2.13) lead to

$$
h(0)>0, \quad h(1)<0 .
$$

Therefore, the desired assertion follows easily from $(2.17)$ and the concavity of $h$ on $(0,1)$ together with Lemma 2.3 .

Case $2 a=\pi / \sqrt{2(4-\pi)}$. Then (2.12), (2.13), (2.15), and (2.16) give

$$
\begin{aligned}
& h(0)=0, \quad h(1)<0, \\
& h^{\prime}(0)>0, \quad h^{\prime}(1)=-\frac{2 \pi^{2}}{\sqrt{2(4-\pi)}}\left[\frac{24-7 \pi}{4-\pi}+3 \sqrt{2(4-\pi)}\right]<0 .
\end{aligned}
$$

From (2.19) and the monotonicity of $h^{\prime}(t)$ on $(0,1)$ we know that there exists $\lambda \in(0,1)$ such that $h(t)$ is increasing on $(0, \lambda]$ and decreasing on $[\lambda, 1)$. Therefore, the desired result follows from (2.18) and the piecewise monotonicity of $h(t)$ on $(0,1)$.

Case $3 a \in(\pi / \sqrt{2(4-\pi)}, \sqrt{\pi+1}+1)$. Then (2.12) and (2.13) imply that

$$
h(0)<0, \quad h(1)<0 .
$$

Therefore, the desired assertion follows from (2.20) and Lemma 2.4 together with the concavity of $h$ on $(0,1)$.

Case $4 a=\sqrt{\pi+1}+1$. Then (2.12), (2.13), (2.15), and (2.16) lead to

$$
h(0)<0, \quad h(1)=0
$$

and (2.19) again holds. From (2.19) and the monotonicity of $h^{\prime}(t)$ on $(0,1)$ we know that there exists $\mu \in(0,1)$ such that $h(t)$ is increasing on $(0, \mu]$ and decreasing on $[\mu, 1)$. Therefore, the desired result follows from (2.21) and the piecewise monotonicity of $h(t)$ on $(0,1)$.

Case $5 a \in(\sqrt{\pi+1}+1, \infty)$. Then (2.12) and (2.13) imply that

$$
h(0)<0, \quad h(1)>0 .
$$

Therefore, the desired assertion follows from Lemma 2.3 and (2.22) together with the concavity of $f$ on $(0,1)$.

\section{Main results}

Theorem 3.1 The following statements are true for all $x>0$ :

(1) if $a \in\left(0,(\sqrt{\pi+1}+1)^{2} / \pi\right]$, then

$$
\frac{a}{\sqrt{x^{2}+2 a}+(a-1) x}<R(x)<\sqrt{\frac{\pi}{a}} \frac{a}{\sqrt{x^{2}+2 a}+(a-1) x} ;
$$

(2) if $a \in[4, \infty)$, then

$$
\sqrt{\frac{\pi}{a}} \frac{a}{\sqrt{x^{2}+2 a}+(a-1) x}<R(x)<\frac{a}{\sqrt{x^{2}+2 a}+(a-1) x} ;
$$


(3) if $a \in\left((\sqrt{\pi+1}+1)^{2} / \pi, 4\right)$, then

$$
\min \left\{1, \sqrt{\frac{\pi}{a}}\right\} \frac{a}{\sqrt{x^{2}+2 a}+(a-1) x}<R(x)<\lambda(a) \frac{a}{\sqrt{x^{2}+2 a}+(a-1) x}
$$

with $\lambda(a)=\left(\sqrt{x_{0}^{2}+2 a}+(a-1) x_{0}\right) R\left(x_{0}\right) / a$, where $x_{0}$ is the unique solution of the equation

$$
\frac{d\left[\left(\sqrt{x^{2}+2 a}+(a-1) x\right) R(x)\right]}{d x}=0
$$

on the interval $(0, \infty)$. In particular, if $a=\pi$, then $x_{0}=0.590 \cdots, \lambda(\pi)=1.011 \cdots$, and

$$
\frac{\pi}{\sqrt{x^{2}+2 \pi}+(\pi-1) x}<R(x)<\frac{\pi \lambda(\pi)}{\sqrt{x^{2}+2 \pi}+(\pi-1) x} .
$$

Proof Let

$$
f_{1}(x)=\frac{a e^{-x^{2} / 2}}{\sqrt{x^{2}+2 a}+(a-1) x}, \quad g(x)=\int_{x}^{\infty} e^{-t^{2} / 2} d t
$$

and $I_{a}(x)$ be defined by (1.6). Then simple computations lead to

$$
\begin{aligned}
& \frac{f_{1}(x)}{g(x)}=\frac{I_{a}(x)}{R(x)}, \\
& f_{1}(\infty)=g(\infty)=0, \\
& \lim _{x \rightarrow 0^{+}} \frac{f_{1}(x)}{g(x)}=\sqrt{\frac{a}{\pi}}, \quad \lim _{x \rightarrow \infty} \frac{f_{1}(x)}{g(x)}=1,
\end{aligned}
$$

and

$$
\frac{f_{1}^{\prime}(x)}{g^{\prime}(x)}=\frac{a\left(a-1+\frac{x}{\sqrt{x^{2}+2 a}}\right)}{\left[\sqrt{x^{2}+2 a}+(a-1) x\right]^{2}}+\frac{a x}{\sqrt{x^{2}+2 a}+(a-1) x} .
$$

Let $t=x / \sqrt{x^{2}+2 a} \in(0,1)$ or $x=\sqrt{2 a} t / \sqrt{1-t^{2}}$. Then (3.8) can be rewritten as

$$
\frac{f_{1}^{\prime}(x)}{g^{\prime}(x)}=\frac{-t^{3}+\left(2 a^{2}-3 a+1\right) t^{2}+(2 a+1) t+(a-1)}{2(1-t+a t)^{2}} .
$$

Differentiating (3.9) gives

$$
\begin{aligned}
\left(\frac{f_{1}^{\prime}(x)}{g^{\prime}(x)}\right)^{\prime} & =\frac{d}{d t}\left[\frac{-t^{3}+\left(2 a^{2}-3 a+1\right) t^{2}+(2 a+1) t+(a-1)}{2(1-t+a t)^{2}}\right] \times \frac{d t}{d x} \\
& =\frac{a(1-t)}{(1-t+a t)^{3}\left(x^{2}+2 a\right)^{3 / 2}} l(t),
\end{aligned}
$$

where

$$
l(t)=(a-1) t^{2}+(a+2) t-2 a^{2}+6 a-1,
$$




$$
l(0)=-2\left(a-\frac{3-\sqrt{7}}{2}\right)\left(a-\frac{3+\sqrt{7}}{2}\right), \quad l(1)=-2 a(a-4) .
$$

Next, we divide our analysis into three cases to determine the sign of $l(t)$ on the interval $(0,1)$.

Case $1 a=1$. We clearly see that $l(t)=3(t+1)>0$ for $t \in(0,1)$.

Case $2 a>1$. It follows from (3.11) that $l(t)$ is strictly convex on $(0,1)$.

We divide the discussions into three subcases.

Subcase $2.1 a \in(1,(3+\sqrt{7}) / 2]$. Then (3.11) and (3.12) lead to $l(t)>l(0) \geq 0$ for $t \in(0,1)$. Subcase $2.2 a \in((3+\sqrt{7}) / 2,4)$. Then (3.12) gives

$$
l(0)<0, \quad l(1)>0 .
$$

It follows from Lemma 2.3 and the convexity of $l(t)$ on $(0,1)$ together with (3.13) that there exists $t_{1} \in(0,1)$ such that $l(t)<0$ for $t \in\left(0, t_{1}\right)$ and $l(t)>0$ for $t \in\left(t_{1}, 1\right)$.

Subcase $2.3 a \in[4, \infty)$. Then (3.12) gives

$$
l(0)<0, \quad l(1) \leq 0 .
$$

Making use of the convexity of $l(t)$ on $(0,1)$ and $(3.14)$ we get

$$
l(t) \leq(1-t) l(0)+t l(1)<0
$$

for $t \in(0,1)$.

Case $30<a<1$. Then from (3.11) we clearly see that $l(t)$ is strictly concave on $(0,1)$.

We divide the discussions into two subcases.

Subcase $3.1 a \in(0,(3-\sqrt{7}) / 2)$. Then (3.12) and Lemma 2.3 lead to the conclusion that (3.13) again holds and there exists $t_{2} \in(0,1)$ such that $l(t)<0$ for $t \in\left(0, t_{2}\right)$ and $l(t)>0$ for $t \in\left(t_{2}, 1\right)$.

Subcase $3.2 a \in[(3-\sqrt{7}) / 2,1)$. Then (3.12) leads to

$$
l(0) \geq 0, \quad l(1)>0 .
$$

Making use of the concavity of $l(t)$ on $(0,1)$ and $(3.15)$ we have

$$
l(t) \geq(1-t) l(0)+t l(1)>0
$$

for $t \in(t, 1)$.

Now, we divide the discussion into three cases to prove the desired results.

Case $A a \in[(3-\sqrt{7}) / 2,(3+\sqrt{7}) / 2]$. Then Subcases 2.1 and 3.2 together with (3.10) and (3.11) lead to the conclusion that $f_{1}^{\prime}(x) / g^{\prime}(x)$ is increasing on $(0, \infty)$. From the monotonicity of $f_{1}^{\prime}(x) / g^{\prime}(x)$ on $(0, \infty)$ and (3.6) together with Lemma 2.1 and the fact that $g^{\prime}(x)=-e^{-x^{2} / 2} \neq$ 0 we know that $f_{1}(x) / g(x)$ is also increasing on $(0, \infty)$. Therefore, (3.1) follows easily from (3.5), (3.7), and the monotonicity of $f_{1}(x) / g(x)$ on $(0, \infty)$.

Case $B a \in[4, \infty)$. Then (3.10) and (3.11) together with Subcase 2.3 lead to the conclusion that $f_{1}^{\prime}(x) / g^{\prime}(x)$ is decreasing on $(0, \infty)$. Making use of (3.6) and Lemma 2.1 together with 
$g^{\prime}(x) \neq 0$ we know that $f_{1}(x) / g(x)$ is also decreasing on $(0, \infty)$. Therefore, (3.2) follows easily from (3.5), (3.7), and the monotonicity of $f_{1}(x) / g(x)$ on $(0, \infty)$.

Case $C a \in(0,(3-\sqrt{7}) / 2) \cup((3+\sqrt{7}) / 2,4)$. Then from Subcases 2.2 and 3.1 we know that there exists $t^{*} \in(0,1)$ such that $l(t)<0$ for $t \in\left(0, t^{*}\right)$ and $l(t)>0$ for $t \in\left(t^{*}, 1\right)$, and (3.10) and (3.11) lead to the conclusion that there exists $x^{*}=\sqrt{2 a} t^{*} / \sqrt{1-t^{* 2}} \in(0, \infty)$ such that $f_{1}^{\prime}(x) / g^{\prime}(x)$ is decreasing on $\left(0, x^{*}\right)$ and increasing on $\left(x^{*}, \infty\right)$.

Note that

$$
\begin{aligned}
H_{f_{1}, g}(x)= & \frac{f_{1}^{\prime}(x)}{g^{\prime}(x)} g(x)-f_{1}(x) \\
= & {\left[\frac{a\left(a-1+\frac{x}{\sqrt{x^{2}+2 a}}\right)}{\left(\sqrt{x^{2}+2 a}+(a-1) x\right)^{2}}+\frac{a x}{\sqrt{x^{2}+2 a}+(a-1) x}\right] \int_{x}^{\infty} e^{-t^{2} / 2} d t } \\
& -\frac{a e^{-x^{2} / 2}}{\sqrt{x^{2}+2 a}+(a-1) x}, \\
H_{f_{1}, g}(0)= & \frac{\sqrt{2 \pi}}{4}\left(\sqrt{a}+\frac{\sqrt{\pi+1}-1}{\sqrt{\pi}}\right)\left(\sqrt{a}-\frac{\sqrt{\pi+1}+1}{\sqrt{\pi}}\right) .
\end{aligned}
$$

We divide the discussion into two subcases.

Subcase $C(1) a \in(0,(3-\sqrt{7}) / 2) \cup((3+\sqrt{7}) / 2,(\pi+2 \sqrt{\pi+1}+2) / \pi]$. Then (3.16) leads to

$$
H_{f_{1}, g}(0) \leq 0
$$

It follows from (3.6), (3.17), and $g^{\prime}(x)=-e^{-x^{2} / 2}<0$ together with the piecewise monotonicity of $f_{1}^{\prime} / g^{\prime}$ and Lemma 2.2 (i) that $f_{1} / g$ is increasing on $(0, \infty)$. Therefore, (3.1) follows easily from (3.5), (3.7), and the monotonicity of $f_{1}(x) / g(x)$ on $(0, \infty)$.

Subcase $C(2) a \in((\pi+2 \sqrt{\pi+1}+2) / \pi, 4)$. Then (3.16) gives

$$
H_{f_{1}, g}(0)>0
$$

From (3.6), (3.18), and $g^{\prime}(x)<0$ together with the piecewise monotonicity of $f_{1}^{\prime} / g^{\prime}$ and Lemma 2.2(ii) we know that there exists $x_{0} \in(0, \infty)$ such that $f_{1} / g$ is decreasing on $\left(0, x_{0}\right)$ and increasing on $\left(x_{0}, \infty\right)$. Consequently, we get

$$
\min \left\{\lim _{x \rightarrow 0} \frac{g(x)}{f_{1}(x)}, \lim _{x \rightarrow \infty} \frac{g(x)}{f_{1}(x)}\right\}<\frac{g(x)}{f_{1}(x)}=\frac{R(x)}{I_{a}(x)}<\frac{R\left(x_{0}\right)}{I_{a}\left(x_{0}\right)}=\lambda(a) .
$$

Therefore, (3.3) follows from (3.7) and (3.19). We clearly see that $x_{0}$ satisfies the equation $\left(f_{1}(x) / g(x)\right)^{\prime}=0$ or $d\left[\left(\sqrt{x^{2}+2 a}+(a-1) x\right) R(x)\right] / d x=0$, and $\lambda(a)=\left(\sqrt{x_{0}^{2}+2 a}+(a-\right.$ 1) $\left.x_{0}\right) R\left(x_{0}\right) / a$.

If $a=\pi$, then numerical computations show that $x_{0}=0.590 \cdots$ and $\lambda(\pi)=1.011 \cdots$.

Theorem 3.2 The following statements are true for all $x>0$ :

(1) if $a \in(0, \pi / \sqrt{2(4-\pi)}]$, then

$$
\frac{a}{\sqrt{x^{2}+\frac{2 a^{2}}{\pi}+\frac{2 a x}{\pi}}}<R(x)<\frac{\pi+2 a}{\pi a} \frac{a}{\sqrt{x^{2}+\frac{2 a^{2}}{\pi}}+\frac{2 a x}{\pi}} ;
$$


(2) if $a \in[\sqrt{\pi+1}+1, \infty)$, then

$$
\frac{\pi+2 a}{\pi a} \frac{a}{\sqrt{x^{2}+\frac{2 a^{2}}{\pi}}+\frac{2 a x}{\pi}}<R(x)<\frac{a}{\sqrt{x^{2}+\frac{2 a^{2}}{\pi}}+\frac{2 a x}{\pi}} ;
$$

(3) if $a \in(\pi / \sqrt{2(4-\pi)}, \sqrt{\pi+1}+1)$, then

$$
\frac{a \theta(a)}{\sqrt{x^{2}+\frac{2 a^{2}}{\pi}}+\frac{2 a x}{\pi}}<R(x)<\max \left\{\frac{\pi+2 a}{\pi a}, 1\right\} \frac{a}{\sqrt{x^{2}+\frac{2 a^{2}}{\pi}}+\frac{2 a x}{\pi}},
$$

where

$$
\theta(a)=\frac{1}{a}\left(\sqrt{x_{0}^{2}+\frac{2 a^{2}}{\pi}}+\frac{2 a x_{0}}{\pi}\right) R\left(x_{0}\right)
$$

and $x_{0}$ is the unique solution of the equation

$$
\frac{d\left[\left(\sqrt{x^{2}+\frac{2 a^{2}}{\pi}}+\frac{2 a x}{\pi}\right) R(x)\right]}{d x}=0
$$

on $(0, \infty)$. In particular, if $a=a_{0}=\pi /(\pi-2)=2.7519 \cdots$, then $x_{0}=1.6108 \cdots, \theta\left(a_{0}\right)=$ $0.9909 \cdots$, and

$$
\frac{\pi \theta\left(a_{0}\right)}{\sqrt{(\pi-2)^{2} x^{2}+2 \pi}+2 x}<R(x)<\frac{\pi}{\sqrt{(\pi-2)^{2} x^{2}+2 \pi}+2 x} .
$$

Proof Let

$$
f_{2}(x)=\frac{a e^{-x^{2} / 2}}{\sqrt{x^{2}+\frac{2 a^{2}}{\pi}}+\frac{2 a x}{\pi}}, \quad g(x)=\int_{x}^{\infty} e^{-t^{2} / 2} d t
$$

and $J_{a}(x)$ be defined by (1.7). Then simple computations lead to

$$
\begin{aligned}
& \frac{f_{2}(x)}{g(x)}=\frac{J_{a}(x)}{R(x)}, \\
& f_{2}(\infty)=g(\infty)=0, \\
& \lim _{x \rightarrow 0^{+}} \frac{f_{2}(x)}{g(x)}=1, \quad \lim _{x \rightarrow \infty} \frac{f_{2}(x)}{g(x)}=\frac{\pi a}{\pi+2 a}, \\
& \frac{f_{2}^{\prime}(x)}{g^{\prime}(x)}=\frac{a x}{\sqrt{x^{2}+\frac{2 a^{2}}{\pi}}+\frac{2 a x}{\pi}}+a \frac{\frac{2 a}{\pi}+\frac{x}{\sqrt{x^{2}+\frac{2 a^{2}}{\pi}}}}{\left(\sqrt{\left.x^{2}+\frac{2 a^{2}}{\pi}+\frac{2 a x}{\pi}\right)^{2}}\right.} \\
& \begin{aligned}
H_{f_{2}, g}(x) & =\frac{f_{2}^{\prime}(x)}{g^{\prime}(x)} g(x)-f_{2}(x) \\
& =\left[\frac{a x}{\sqrt{x^{2}+\frac{2 a^{2}}{\pi}}+\frac{2 a x}{\pi}}+a \frac{\frac{2 a}{\pi}+\frac{x}{\sqrt{x^{2}+\frac{2 a^{2}}{\pi}}}}{\left(\sqrt{x^{2}+\frac{2 a^{2}}{\pi}+\frac{2 a x}{\pi}}\right)^{2}}\right] \int_{x}^{\infty} e^{-t^{2}} d t-\frac{a e^{-x^{2} / 2}}{\sqrt{x^{2}+\frac{2 a^{2}}{\pi}}+\frac{2 a x}{\pi}}
\end{aligned}
\end{aligned}
$$




$$
H_{f_{2}, g}(0)=H_{f_{2}, g}(\infty)=0 \text {. }
$$

Let $t=x / \sqrt{x^{2}+2 a^{2} / \pi} \in(0,1)$ or $x=\sqrt{2} a t / \sqrt{\pi\left(1-t^{2}\right)}$. Then (3.26) becomes

$$
\frac{f_{2}^{\prime}(x)}{g^{\prime}(x)}=-\frac{\pi}{2 a} \frac{\pi^{2} t^{3}+\left(2 \pi a-4 a^{3}\right) t^{2}-\left(2 \pi a^{2}+\pi^{2}\right) t-2 \pi a}{(\pi+2 a t)^{2}} .
$$

Differentiating (3.28) gives

$$
\begin{aligned}
\left(\frac{f_{2}^{\prime}(x)}{g^{\prime}(x)}\right)^{\prime}= & -\frac{\pi}{2 a} \frac{d}{d t}\left(\frac{\pi^{2} t^{3}+\left(2 \pi a-4 a^{3}\right) t^{2}-\left(2 \pi a^{2}+\pi^{2}\right) t-2 \pi a}{(\pi+2 a t)^{2}}\right) \times \frac{d t}{d x} \\
= & -\frac{\pi}{2 a}\left(\pi \frac{2 \pi a t^{3}+3 \pi^{2} t^{2}+\left(6 \pi a-4 a^{3}\right) t+\left(8 a^{2}-\pi^{2}-2 \pi a^{2}\right)}{(\pi+2 a t)^{3}}\right) \\
& \times \frac{2 a^{2} \sqrt{\pi}}{\left(\pi x^{2}+2 a^{2}\right)^{3 / 2}} \\
= & \frac{a \pi^{5 / 2}}{(\pi+2 a t)^{3}\left(2 a^{2}+\pi x^{2}\right)^{3 / 2}} h(t),
\end{aligned}
$$

where $h(t)$ is defined by Lemma 2.4 .

We divide the proof into three cases.

Case $1 a \in(0, \pi / \sqrt{2(4-\pi)}]$. Then from Lemma 2.5(i) and (3.29) we know that there exists $x_{1}=\sqrt{2} a t_{1} / \sqrt{\pi\left(1-t_{1}\right)^{2}} \in(0, \infty)$ such that $f_{2}^{\prime} / g^{\prime}$ is increasing on $\left(0, x_{1}\right)$ and decreasing on $\left(x_{1}, \infty\right)$. It follows from the piecewise monotonicity of $f_{2}^{\prime} / g^{\prime},(3.24),(3.27)$, $g^{\prime}(x)=-e^{-x^{2} / 2}<0$, and Lemma $2.2(\mathrm{i})$ that $f_{2} / g$ is decreasing on $(0, \infty)$. Therefore, $(3.20)$ follows from (3.23) and (3.25) together with the monotonicity of $f_{2} / g$.

Case $2 a \in[\sqrt{\pi+1}+1, \infty)$. Then from Lemma 2.5(iii) and (3.29) we know that there exists $x_{1}^{*}=\sqrt{2} a t_{1}^{*} / \sqrt{\pi\left(1-t_{1}^{*}\right)^{2}} \in(0, \infty)$ such that $f_{2}^{\prime} / g^{\prime}$ is decreasing on $\left(0, x_{1}^{*}\right)$ and increasing on $\left(x_{1}^{*}, \infty\right)$. It follows from the piecewise monotonicity of $f_{2}^{\prime} / g^{\prime},(3.24),(3.27), g^{\prime}(x)<0$, and Lemma $2.2(\mathrm{i})$ that $f_{2} / g$ is increasing on $(0, \infty)$. Therefore, $(3.21)$ follows from $(3.23)$ and (3.25) together with the monotonicity of $f_{2} / g$.

Case $3 a \in(\pi / \sqrt{2(4-\pi)}, \sqrt{\pi+1}+1)$. Then from Lemma 2.5(ii) and (3.29) together with $g>0$ and $\left(H_{f_{2}, g}\right)^{\prime}=\left(f_{2}^{\prime} / g^{\prime}\right)^{\prime} g$ we know that there exists $x_{11}=\sqrt{2} a t_{11} / \sqrt{\pi\left(1-t_{11}\right)^{2}}, x_{12}=$ $\sqrt{2} a t_{12} / \sqrt{\pi\left(1-t_{12}\right)^{2}} \in(0, \infty)$ with $x_{11}<x_{12}$ such that $H_{f_{2}, g}$ is decreasing on $\left(0, x_{11}\right) \cup$ $\left(x_{12}, \infty\right)$ and increasing on $\left(x_{11}, x_{12}\right)$.

Making use of the piecewise monotonicity of $H_{f_{2}, g}$ and (3.27) we conclude that there exists $x_{0} \in(0, \infty)$ such that $H_{f_{2}, g}(x)<0$ for $x \in\left(0, x_{0}\right)$ and $H_{f_{2}, g}(x)>0$ for $x \in\left(x_{0}, \infty\right)$, then the identity $\left(f_{2} / g\right)^{\prime}=g^{\prime} H_{f_{2}, g} / g^{2}$ and $g^{\prime}<0$ lead to the conclusion that $f_{2} / g$ is increasing on $\left(0, x_{0}\right)$ and decreasing on $\left(x_{0}, \infty\right)$. Therefore, (3.22) follows easily from (3.23) and (3.25) together with the piecewise monotonicity of $f_{2} / g$, where

$$
\theta(a)=\frac{R\left(x_{0}\right)}{J_{a}\left(x_{0}\right)}=\frac{1}{a}\left(\sqrt{x_{0}^{2}+\frac{2 a^{2}}{\pi}}+\frac{2 a x_{0}}{\pi}\right) R\left(x_{0}\right) .
$$

We clearly see that $x_{0}$ satisfies the equation $\left(f_{2} / g\right)^{\prime}=0$, namely $x_{0}$ is the unique solution of the equation

$$
\frac{d\left[\left(\sqrt{x^{2}+\frac{2 a^{2}}{\pi}}+\frac{2 a x}{\pi}\right) R(x)\right]}{d x}=0
$$


on $(0, \infty)$. In particular, if $a=a_{0}=\pi /(\pi-2)=2.7519 \cdots$, then numerical computations show that $x_{0}=1.6108 \cdots$ and $\theta\left(a_{0}\right)=0.9909 \cdots$.

Remark 3.1 Let $x>0$, and $I_{a}(x)$ and $J_{a}(x)$ be defined by (1.6) and (1.7), respectively. Then the functions $a \rightarrow I_{a}(x)$ and $a \rightarrow J_{a}(x)$ are increasing on $(0, \infty)$, and the functions $a \rightarrow$ $\sqrt{\pi / a} I_{a}(x)$ and $a \rightarrow(\pi+2 a) J_{a}(x) /(\pi a)$ are decreasing on $(0, \infty)$ due to

$$
\begin{aligned}
& \frac{\partial I_{a}(x)}{\partial a}=\frac{x^{2}+a-x \sqrt{x^{2}+2 a}}{\sqrt{x^{2}+2 a}+\left(\sqrt{x^{2}+2 a}+(a-1) x\right)^{2}}>0, \\
& \frac{\partial}{\partial a}\left(\sqrt{\frac{\pi}{a}} I_{a}(x)\right)=-\frac{1}{2} \sqrt{\frac{\pi}{a}} x \frac{(1+a) \sqrt{x^{2}+2 a}-x}{\sqrt{x^{2}+2 a}\left(\sqrt{x^{2}+2 a}+(a-1) x\right)^{2}}<0, \\
& \frac{\partial J_{a}(x)}{\partial a}=\frac{x^{2}}{\sqrt{x^{2}+2 a^{2} / \pi}\left(\sqrt{x^{2}+2 a^{2} / \pi}+2 a x / \pi\right)^{2}}>0, \\
& \frac{\partial}{\partial a}\left(\frac{\pi+2 a}{\pi a} J_{a}(x)\right)=-\frac{2}{\pi} \frac{x\left(\sqrt{x^{2}+2 a^{2} / \pi}-x\right)+a}{\sqrt{x^{2}+2 a^{2} / \pi}\left(\sqrt{x^{2}+2 a^{2} / \pi}+2 a x / \pi\right)^{2}}<0 .
\end{aligned}
$$

From Theorems 3.1 and 3.2, and their proofs together with Remark 3.1 we get Corollary 3.1 .

Corollary 3.1 Let $a_{1}, b_{1}, a_{2}, b_{2}>0$. Then the double inequalities

$$
\frac{a_{1}}{\sqrt{x^{2}+2 a_{1}}+\left(a_{1}-1\right) x}<R(x)<\frac{b_{1}}{\sqrt{x^{2}+2 b_{1}}+\left(b_{1}-1\right) x}
$$

and

$$
\frac{a_{2}}{\sqrt{x^{2}+2 a_{2}^{2} / \pi}+2 a_{2} x / \pi}<R(x)<\frac{b_{2}}{\sqrt{x^{2}+2 b_{2}^{2} / \pi}+2 b_{2} x / \pi}
$$

hold for all $x>0$ if and only if $a_{1} \leq \pi, b_{1} \geq 4, a_{2} \leq \pi / \sqrt{2(4-\pi)}=2.3976 \cdots$, and $b_{2} \geq$ $\pi /(\pi-2)=2.7516 \cdots$.

Remark 3.2 Letting $a_{1}=\pi, b_{1}=4, a_{2}=\pi / \sqrt{2(4-\pi)}$, and $b_{2}=\pi /(\pi-2)$. Then (3.30) and (3.31) lead to

$$
W_{1,2}(x)<R(x)<W_{0,3}(x), \quad W_{3,0}(x)<R(x)<W_{2,1}(x)
$$

for all $x>0$, which implies inequality (1.3), where $W_{3,0}(x), W_{1,2}(x), W_{2,1}(x)$, and $W_{0,3}(x)$ are defined by (1.4) and (1.5).

Letting $a=0^{+}, 1,2,(\sqrt{\pi+1}+1)^{2} / \pi$ in Theorem 3.1(1), $a=4,5, \infty$ in Theorem 3.1(2), $a=0^{+}, 1,2, \pi / \sqrt{2(4-\pi)}$ in Theorem 3.2(1) and $a=\sqrt{\pi+1}+1, \pi, 4, \infty$ in Theorem 3.2(2), respectively. Then we get Corollary 3.2 immediately. 
Corollary 3.2 The following inequalities for the Mills ratio $R(x)$ :

$$
\begin{aligned}
& \frac{x}{x^{2}+1}<\frac{1}{\sqrt{x^{2}+2}}<\frac{2}{\sqrt{x^{2}+4}+x}<\frac{(\sqrt{\pi+1}+1)^{2}}{\sqrt{\pi^{2} x^{2}+2 \pi(\sqrt{\pi+1}+1)^{2}}+2(\sqrt{\pi+1}+1) x} \\
& <R(x)<\frac{\pi(\sqrt{\pi+1}+1)}{\sqrt{\pi^{2} x^{2}+2 \pi(\sqrt{\pi+1}+1)^{2}}+2(\sqrt{\pi+1}+1) x}<\frac{\sqrt{2 \pi}}{\sqrt{x^{2}+4}+x}<\frac{\sqrt{\pi}}{\sqrt{x^{2}+2}}, \\
& \frac{\sqrt{5 \pi}}{\sqrt{x^{2}+10}+4 x}<\frac{2 \sqrt{\pi}}{\sqrt{x^{2}+8}+3 x}<R(x)<\frac{4}{\sqrt{x^{2}+8}+3 x}<\frac{5}{\sqrt{x^{2}+10}+4 x}<\frac{1}{x}, \\
& \frac{\pi}{\sqrt{\pi^{2} x^{2}+2 \pi}+2 x}<\frac{2 \pi}{\sqrt{\pi^{2} x^{2}+8 \pi}+4 x}<\frac{\pi}{\sqrt{2(4-\pi) x^{2}+2 \pi}+2 x}<R(x) \\
& <\frac{\sqrt{2(4-\pi)}+2}{\sqrt{2(4-\pi) x^{2}+2 \pi}+2 x}<\frac{\pi+4}{\sqrt{\pi^{2} x^{2}+8 \pi}+4 x} \\
& <\frac{\pi+2}{\sqrt{\pi^{2} x^{2}+2 \pi}+2 x}<\frac{1}{x} \text {, } \\
& \frac{2}{2 x+\sqrt{2 \pi}}<\frac{\pi+8}{\sqrt{\pi^{2} x^{2}+32 \pi}+8 x}<\frac{3}{\sqrt{x^{2}+2 \pi}+2 x} \\
& <\frac{(\sqrt{\pi+1}+1)^{2}}{\sqrt{\pi^{2} x^{2}+2 \pi(\sqrt{\pi+1}+1)^{2}}+2(\sqrt{\pi+1}+1) x}<R(x) \\
& <\frac{\pi(\sqrt{\pi+1}+1)}{\sqrt{\pi^{2} x^{2}+2 \pi(\sqrt{\pi+1}+1)^{2}}+2(\sqrt{\pi+1}+1) x} \\
& <\frac{\pi}{\sqrt{x^{2}+2 \pi}+2 x}<\frac{4 \pi}{\sqrt{\pi^{2} x^{2}+32 \pi}+8 x}<\frac{\pi}{2 x+\sqrt{2 \pi}}
\end{aligned}
$$

hold for all $x>0$.

\section{Competing interests}

The authors declare that they have no competing interests.

\section{Authors' contributions}

All authors contributed equally to the writing of this paper. All authors read and approved the final manuscript.

\section{Author details}

School of Mathematics and Computation Science, Hunan City University, Yiyang, 413000, China.

\section{Acknowledgements}

The research was supported by the Natural Science Foundation of China under Grants 61374086 and 11171307, and the Natural Science Foundation of Zhejiang Province under Grant LY13A010004.

Received: 24 June 2015 Accepted: 20 August 2015 Published online: 17 September 2015

\section{References}

1. Mills, JP: Table of the ratio: area to bounding ordinate, for any portion of normal curve. Biometrika 18(3-4), 395-400 (1926)

2. Laplace, PS: Traité de Mécanique Céleste, Tome IV. Chez Courcier, Paris (1805)

3. Gordon, RD: Values of Mills' ratio of area to bounding ordinate and of the normal probability integral for large values of the argument. Ann. Math. Stat. 12, 364-366 (1941)

4. Birnbaum, ZW: An inequality for Mill's ratio. Ann. Math. Stat. 13, 245-246 (1942)

5. Komatu, Y: Elementary inequalities for Mills ratio. Rep. Stat. Appl. Res. UJSE 4, 69-70 (1955)

6. Sampford, MR: Some inequalities on Mill's ratio and related functions. Ann. Math. Stat. 24, 130-132 (1953)

7. Shenton, LR: Inequalities for the normal integral including a new continued fraction. Biometrika 41, 177-189 (1954)

8. Pollak, HO: A remark on 'Elementary inequalities for Mills' ratio' by Yûsaku Komatu. Rep. Stat. Appl. Res. UJSE 4, 110 (1956) 
9. Boyd, AV: Inequalities for Mills' ratio. Rep. Stat. Appl. Res. UJSE 6, 44-46 (1959)

10. Gasull, A, Utzet, F: Approximating Mills ratio. J. Math. Anal. Appl. 420(2), 1832-1853 (2014)

11. Gautschi, W: Some elementary inequalities relating to the gamma and incomplete gamma function. J. Math. Phys. 38, 77-81 (1959/1960)

12. Mitrinović, DS: Analytic Inequalities. Springer, New York (1970)

13. Börjesson, $P O$, Sundberg, C-EW: Simple approximations of the error function $Q(x)$ for communications applications IEEE Trans. Commun. 27(3), 639-643 (1979)

14. Alzer, H: On some inequalities for incomplete gamma function. Math. Comput. 66(218), 771-778 (1997)

15. Pinelis, I: Monotonicity properties of the relative error of a Padé approximation for Mills' ratio. JIPAM. J. Inequal. Pure Appl. Math. 3(2), 20 (2002)

16. Karagiannidis, GK, Lioumpas, AS: An improved approximation for the Gaussian Q-function. IEEE Commun. Lett. 11(8), 644-646 (2007)

17. Aludaat, KM, Alodat, MT: A note on approximating the normal distribution function. Appl. Math. Sci. 2(9-12), 425-429 (2008)

18. Baricz, Á: Mills' ratio: monotonicity patterns and functional inequalities. J. Math. Anal. Appl. 340(2), 1362-1370 (2008)

19. Baricz, Á: Mills' ratio: reciprocal convexity and functional inequalities. Acta Univ. Sapientiae Math. 4(1), 26-35 (2012)

20. Choudhury, A: A simple approximation to the area under standard normal curve. Math. Stat. 2(3), 147-149 (2014)

21. Alessandro, S, Emanuela, E: Very simply explicitly invertible approximations of normal cumulative and normal quantile function. Appl. Math. Sci. 8(87), 4323-4341 (2014)

22. Small, CG: Expansions and Asymptotics for Statistics. CRC Press, Boca Raton (2010)

23. Pinelis, I: I'Hospital type results for monotonicity, with applications. JIPAM. J. Inequal. Pure Appl. Math. 3(1), 5 (2002)

24. Pinelis, I: I'Hospital type rules for oscillation, with applications. JIPAM. J. Inequal. Pure Appl. Math. 2(3), 33 (2001)

25. Yang, Z-H: A new way to prove I'Hospital monotone rules with applications. arXiv:1409.6408 [math. CA]

\section{Submit your manuscript to a SpringerOpen ${ }^{\circ}$ journal and benefit from:}

- Convenient online submission

Rigorous peer review

- Immediate publication on acceptance

- Open access: articles freely available online

- High visibility within the field

- Retaining the copyright to your article 